\title{
Calcific shoulder periarthritis (tendinitis) in adult onset diabetes mellitus: a controlled study
}

\author{
M E MAVRIKAKIS, S DRIMIS, D A KONTOYANNIS, A RASIDAKIS, \\ E S MOULOPOULOU, AND S KONTOYANNIS
}

From the Department of Clinical Therapeutics, University of Athens, Alexandra General Hospital, GR-115 28 Athens, Greece

SUMmaRY Two groups, one of 824 adult diabetics and one of 320 age and sex matched non-diabetics, were examined for abnormal glucose metabolism and calcifications on anteroposterior shoulder $x$ rays. Two hundred and sixty two $(31.8 \%)$ of the diabetics had shoulder calcification compared with $33(10 \cdot 3 \%)$ of the control group, with a preponderant localisation in the right shoulder. Diabetes of long duration treated with insulin for a long time was associated with a larger percentage of shoulder calcifications. These data and previous laboratory findings suggest a possible pathogenetic correlation between the prevalence of calcific shoulder tendinitis and diabetes.

Diabetes is a common disease, affecting about $6 \%$ of the Athens population, and generally making the patient susceptible to a wide range of metabolic disturbances. ${ }^{1}$ A variety of rheumatological syndromes, such as osteopenia, gout, pseudogout, neuroarthropathy, osteolysis, carpal tunnel syndrome, osteoarthrosis, Dupuytren's contracture, cheiroarthropathy, diffuse idiopathic skeletal hyperostosis, and periarthritis, may all present in association with diabetes mellitus. Only neuroarthropathy and osteolysis are definite associations, the others being considered as probable or even possible associations. $^{2}$

Periarthritis is a term which refers to inflammatory disorders of structures surrounding joints - that is, tendons, tendon sheaths, capsules, and bursae. Publications discussing the relation between diabetes mellitus and periarthritis are few. Only three controlled studies have been published. Two suggest a four- to sixfold increased prevalence of association between frozen shoulder and diabetes ${ }^{34}$ whereas the third records calcifications of the shoulders in a small group of diabetics. ${ }^{5}$

The purpose of this controlled study was to investigate the prevalence of calcific shoulder tendinitis in a large group of adult diabetics and to report

Accepted for publication 19 July 1988.

Correspondence to Dr M E Mavrikakis, Department of Clinical Therapeutics, University of Athens School of Medicine, Alexandra General Hospital, Vas. Sofias 80, GR-115 28 Athens, Greece. some clinical interrelations between these two entities.

\section{Patients and methods}

Eight hundred and twenty four consecutive adult diabetics (340 men, 484 women with type II diabetes) and 320 cases of age and sex matched nondiabetics (129 men, 191 women-the control group) were studied. The diabetic group ranged in age from 36 to 92 years (mean 66.1) and the control group from 34 to 86 years (mean 65.7).

The diagnosis of diabetes mellitus was based on the clinical history and the abnormal fasting blood sugar values. The control group comprised subjects without a family history of diabetes, with normal fasting blood sugar values, and with normal sugar values two hours after a meal.

All diabetics and controls were inpatients from an internal university medicine department.

For the diabetics the following were recorded: duration of the disease from diagnosis to enrolment in the study, details of antidiabetic drugs used (oral or parenteral insulin), and duration of the insulin administration.

Standard anteroposterior $x$ ray examinations of both shoulders were made for all diabetics and controls. The films were evaluated 'blindly' by the same two observers. In addition, for diabetics and controls whose shoulder $x$ rays showed calcifications a detailed clinical medical history of their symptoms and signs (pain in the shoulders either during the 
study or previously) was obtained and the range of movement of the shoulders was evaluated. In the group of diabetics with calcific shoulder periarthritis the longitudinal axis of shoulder calcifications was measured, and a neurological examination was made in both groups. Subjects with clinical signs of peripheral neuropathy or evidence of cerebrovascular episodes, post infarction shoulder-hand syndrome, and those with known injuries or fractures in the region of shoulders and renal impairment were excluded from both groups. Serum urea, creatinine, calcium, and phosphorus were determined in diabetics and controls. Statistical analysis of the results was by $\chi^{2}$ test and Student's $t$ test.

\section{Results}

Unilateral or bilateral calcific shoulder periarthritis was found in $262 / 824(31.8 \%)$ diabetics and in $33 / 320(10 \cdot 3 \%)$ of controls-a significant difference $\left(\chi^{2}=55 \cdot 5, \mathrm{p}<0.001\right)$.

Calcific shoulder periarthritis in diabetics was located in the right shoulder in $117 / 262(44.7 \%)$, in the left shoulder in $70 / 262(26.7 \%)$, and bilaterally in $75 / 262(28.6 \%)$, while in the control group the respective locations were $12 / 33(36 \%)$, $9 / 33(27 \%)$, and $12 / 33(36 \%)$ in the right shoulder, left shoulder, and bilaterally $\left(\chi^{2}=3.64, p<0.05 ; \chi^{2}=4.6, p<0.05\right.$; $\chi^{2}=0.8, p<0.05$ respectively).

Eighty four of 262 diabetics with calcific shoulder periarthritis $(32.1 \%)$ had received insulin for a mean duration of 7.4 (SE 1.3) years (range 1-10), while $105 / 562(18.7 \%)$ of diabetics without calcific $\Rightarrow$ shoulder periarthritis $\left(\chi^{2}=18.09, \mathrm{p}<0.001\right)$ had re- $\stackrel{\oplus}{\stackrel{\rho}{+}}$ ceived insulin for a mean duration of $4.2(0.9)$ years?

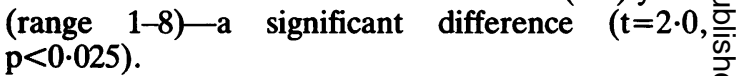

The mean duration of diabetes mellitus from $\propto$ diagnosis for diabetics with calcific shoulder periar-» thritis was 11.5 (SE 2.0) years (range 3-35) and for $\overrightarrow{0}$ the diabetics without calcific shoulder periarthritis:$6.2(0.9)$ years $(t=2.4, p<0.01)$.

The mean age of diabetics with calcific shoulder periarthritis was $66.0(6.4)$ years (range 36-92), of $\frac{2}{2}$ diabetics without calcific shoulder periarthritis $66.2+$ (6.1) years (range 38-88), of controls with calcific $c_{\omega}^{\infty}$ shoulder tendinitis 65.5 (5.4) years (range 37-89), iv and of controls without calcific shoulder periarthritis $65 \cdot 8(6 \cdot 2)$ years (range 34-86)-no significant differ-은 ence.

The size of calcifications (longitudinal axis) in the 3 group of diabetics ranged from $3 \mathrm{~mm}$ to $22 \mathrm{~mm}$.

Among the 262 diabetics with calcific shoulder periarthritis, $85(32.4 \%)$ were symptomatic either $\overrightarrow{0}$ before or during the study (pain, limitation of rangece of movement of the calcified shoulders). Diabetics with calcific shoulder periarthritis were symptomatic when the size of calcification was more than $16 \mathrm{~mm}$ in the longitudinal axis.

Table 1 shows the serum mean values (SE) of urea, creatinine, calcium, and phosphorus for diabetics and controls with or without calcific shouldero periarthritis. No significant difference was found between any group for any of these serum values

Table 1 Mean serum values, standard deviations, and standard errors of urea, calcium, phosphorus, and creatinine in diabetics and controls with and without calcific shoulder periarthritis

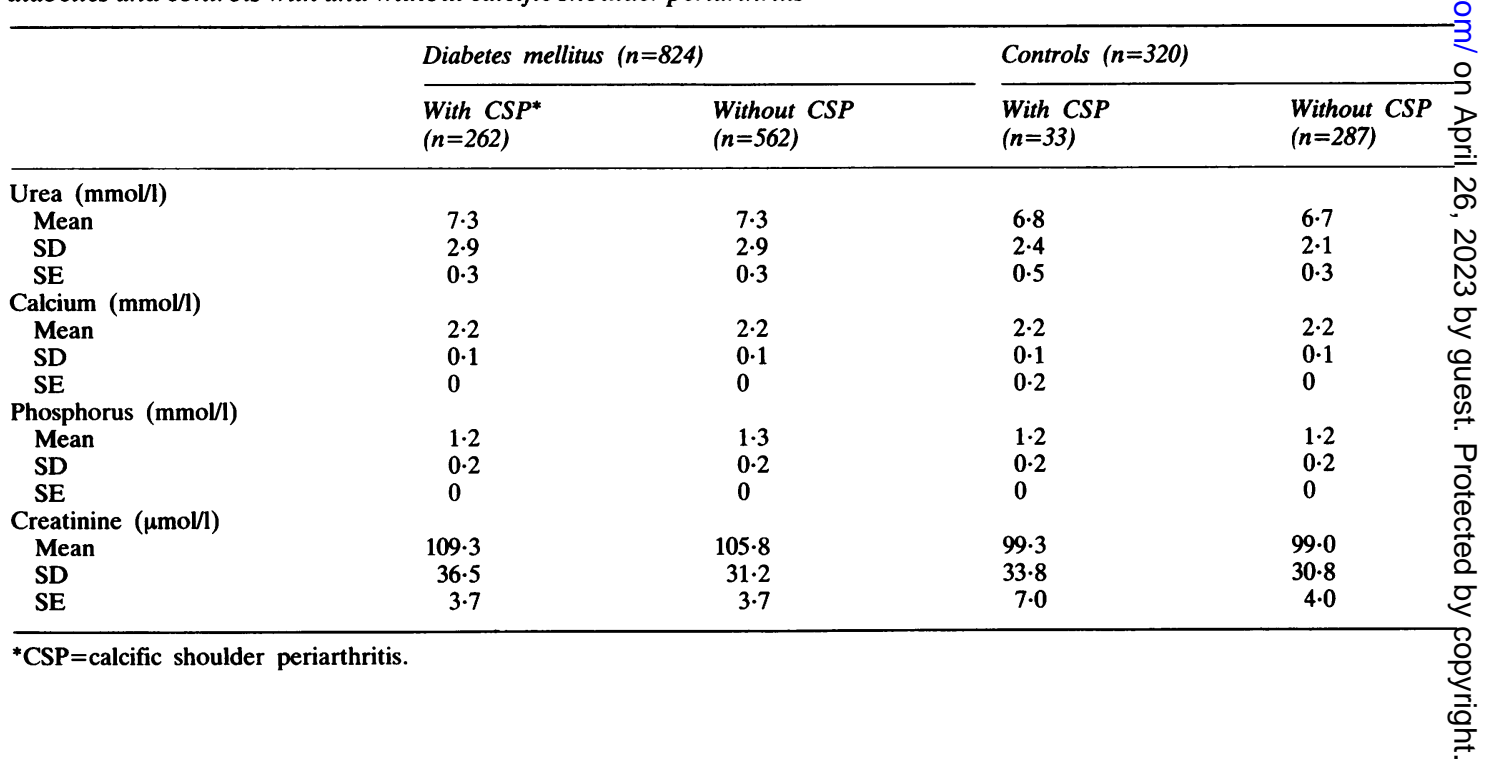




\section{Discussion}

This controlled study reports that calcific shoulder periarthritis is three times more prevalent in diabetics than in a non-diabetic control group. The increased prevalence of calcific shoulder tendinitis in diabetics suggests that the possibility of unsuspected or subclinical diabetes mellitus should be excluded in cases where shoulder calcifications are found by chance, especially if there is a positive family history of diabetes mellitus. In this study the increased prevalence of calcific shoulder tendinitis in diabetics could not be correlated with the age or presence of osteoarthrosis as there was no difference in mean age of diabetics and controls, as shoulder osteoarthrosis is not common, ${ }^{6}$ and additionally, because some patients showed radiologically degenerative changes of the shoulders without calcifications.

The greater number of calcifications in the right shoulder may be related not only to diabetes mellitus but also to the fact that most of the population is right handed and thus there is increased risk of microtraumas in this shoulder, which may be responsible for shoulder tendinitis. ${ }^{7}$

A close relation between diabetes mellitus and calcific shoulder tendinitis is indicated by the finding that the mean duration of diabetes mellitus in diabetics with calcific tendinitis was significantly longer than in the diabetics without calcification, and that a higher percentage of diabetics with calcific shoulder periarthritis had been treated with insulin and for a longer period of time.

It is well known that in longstanding and poorly controlled diabetes mellitus angiopathy is more common and severe. Experimental studies have shown that decreased blood flow in the shoulders facilitates and precipitates the calcification of the surrounding tendons. 89

In addition, studies in tissue cultures and in cultures of fibroblasts from diabetics have shown that insulin deficiency inhibits collagen synthesis ${ }^{10}$ as well as producing an early senescence of fibroblasts, ${ }^{11}$ mechanisms which may contribute to calcification of the connective tissue. ${ }^{7}$

Furthermore, defective formation of glycosaminoglycan is suggested by the work of Schiller and Dröfman, who showed that insulin was necessary for the production of glycosaminoglycan from glucose. ${ }^{12}$ In rats made diabetic by streptozocin the enzymes responsible for degradation of glycosaminoglycans were increased, an effect reversed by the transplantation of pancreatic cells. ${ }^{13}$ In addition, in such rats 24 hour glycosaminoglycan excretion was increased and correlated positively with the duration of experimental diabetes mellitus. A significant reduction in the articular and periarticular tissues was shown by the alcian blue stain. ${ }^{14}{ }^{15}$ It is well known that chains of chondroitin sulphate bind $\mathrm{Ca}^{++}$ions ${ }^{16}$ and that endochondral calcification is accompanied by a decrease in glycosaminoglycan content of the tissue. ${ }^{17}$

It appears possible that apart from these interrelations between diabetes mellitus and calcific shoulder periarthritis other factors, perhaps genetic or immunological, have at least some role in the pathogenesis of calcific shoulder periarthritis in adult diabetics. ${ }^{18} 19$

Of the diabetics with calcific tendinitis of the shoulder, $32.4 \%$ were symptomatic, which is in accordance with the study of Simon, who reported a similar percentage $(34.5 \%)$ in calcified shoulder periarthritis not related to diabetes. ${ }^{20}$

In conclusion, it appears that calcific shoulder periarthritis has a high prevalence among adult onset diabetics, and that an interrelation exists between diabetes mellitus and calcific tendinitis of the shoulders. Many factors may play a part in the pathogenesis of calcification in diabetes mellitus, and as diabetes mellitus is a multifactorial disease calcific shoulder periarthritis may also represent a multifactorial entity.

\section{References}

1 Holt L P. Rheumatological manifestations of diabetes mellitus. Clin Rheum Dis 1981; 7: 723-46.

2 Pastan R, Cohen S A. The rheumatologic manifestations of diabetes mellitus. Med Clin North Am 1978; 62: 829-39.

3 Bridgman J F. Periarthritis of the shoulder and diabetes mellitus. Ann Rheum Dis 1972; 31: 69-71.

4 Lequesne $M$, Dang $N$, Bensasson $M$, Merry C. Increased association of diabetes mellitus with capsulitis of the shoulder and shoulder hand syndrome. Scand J Rheumatol 1977; 6: 53-6.

5 Kaklamanis P, Rigas A, Gianatos J, et al. Calcification of the shoulder and diabetes mellitus. $N$ Engl $J$ Med 1975; 293: 1266-7.

6 Neer C. Reconstructive surgery and rehabilitation of the shoulder. In: Kelley W N, Harris E D Jr, Ruddy S, Sledge C B, eds. Textbook of rheumatology. Philadelphia, London, Toronto: Saunders 1981: 1944-59.

7 Bywaters L G E. Lesions of bursae, tendons and tendons sheaths. Clin Rheum Dis 1979; 5: 883.

8 Macnab I. Rotator cuff tendinitis. Ann R Col Surg Engl 1973; 53: 271-87.

9 Luben B A, Sherman J K, Wadkins C L. In: McCarthy D J, ed Arthritis and allied conditions. 9th ed. Philadelphia: Lea and Febiger, 1979: 1098.

10 Canalis E M, Dietrich J W, Maini D M, Raisz L G. Hormonal control of bone collagen synthesis in vitro. Effects of insulin and glucogen. Endocrinology 1977; 100: 668-74.

11 Goldstein S, Littlefield J W, Soeldner J S. Diabetes mellitus and ageing. Diminished plating eficiency of culture human fibroblasts. Proc Natl Acad Sci USA 1969; 64: 158-60.

12 Schiller S, Dröfman A. The metabolism of mucopolysaccharides in animals. The influence of insulin. J Biol Chem 1957; 227: 625-32.

13 Silberberg R, Hirschberg C E, Lesker P. Enzymes studied in 
214 Mavrikakis, Drimis, Kontoyannis, Rasidakis, Moulopoulou, Kontoyannis

the articular cartilage of diabetic rats and of rats transplanted pancreatic islets. Diabetes $1977 ; 26$ : 723-35.

14 Mavrikakis $M$. Calcific shoulder periarthritis in diabetes mellitus. A clinical and experimental study. Athens University 1986. (Thesis.)

15 Mavrikakis M, Karli I, Kittas Ch, et al. Determination of glucosaminoglycans of the urine and the articular and periarticular tissues in experimental diabetes induced by streptozotocin. Iatriki 1988; 54: 189-93.

16 Chadially F N. Fine structure of joints. In: Sokoloff L, ed. The joints of synovial fluid. New York: Academic Press, 1978: 140.
17 Hynes $R$ O. Cell surface proteins and malignant transformation. Biochim Biophys Acta 1976; 458: 73-6.

18 Zervas J, Mavrikakis M, Kehayoglou K, et al. HLA antigens in diabetics with calcific shoulder periarthritis. Clin Exp Rheumatol 1986; 4: 351-3.

19 Mavrikakis M, Baltopoulos G, Kehayoglou K, et al. Serum immunoglobulin levels in diabetic patients with calcific shoulder $\frac{\mathbb{Q}}{\Omega}$ periarthritis. IRCS Medical Science 1984; 12 : 108.

20 Simon W. Soft tissue disorders of the shoulder, frozen shoulder, $\mathcal{G}$ calcific tendinitis and bicipital tendinitis. Orthop Clin North Am $\overrightarrow{0}$ 1975; 6: 521-9. 